\title{
Uncoupling Protein, UCP-4 May Be Involved in Neuronal Defects During Aging and Resistance to Pathogens in Caenorhabditis elegans
}

\author{
Injeong Cho ${ }^{1,2}$, Gyu Jin Hwang ${ }^{1,2}$, and Jeong Hoon Cho ${ }^{1, *}$
}

\begin{abstract}
Uncoupling proteins (UCPs) are mitochondrial inner membrane proteins that function to dissipate proton motive force and mitochondrial membrane potential. One UCP has been identified in Caenorhabditis elegans (C. elegans), namely UCP-4. In this study, we examined its expression and localization using a GFP marker in C. elegans. ucp-4 was expressed throughout the body from early embryo to aged adult and UCP-4 was localized in the mitochondria. It is known that increased mitochondrial membrane protential leads to a reactive oxygen species (ROS) increase, which is associated with age-related diseases, including neurodegenerative diseases in humans. A ucp-4 mutant showed increased mitochondrial membrane protential in association with increased neuronal defects during aging, and the neurons of ucp-4 overexpressing animals showed decreased neuronal defects during aging. These results suggest that UCP-4 may be involved in neuroprotection during aging via relieving mitochondrial membrane protential. We also investigated the relationship between UCP-4 and innate immunity because increased ROS can affect innate immunity. ucp-4 mutant displayed increased resistance to the pathogen Staphylococcus aureus compared to wild type. The enhanced immunity in the ucp-4 mutant could be related to increased mitochondrial membrane protential, presumably followed by increased ROS. In summary, UCP-4 might have an important role in neuronal aging and innate immune responses through mediating mitochondrial membrane protential.
\end{abstract}

\section{INTRODUCTION}

Uncoupling proteins (UCPs) are a small family of mitochondrial carrier proteins with five identified members in mammalian

\footnotetext{
${ }^{1}$ Department of Biology Education, College of Education, Chosun University, Gwangju 61452, Korea, ${ }^{2}$ These authors contributed equally to this work

*Correspondence: renocho@chosun.ac.kr
}

Received 13 May, 2016; revised 19 July, 2016; accepted 1 August, 2016; published online 20 September, 2016

Keywords: $C$. elegans, innate immune, mitochondrial membrane potential $\left(\psi_{\mathrm{m}}\right)$, neuronal defects, $u c p-4$ systems. The first discovered UCP1 was localized in brown adipose tissue and was shown to dissipate the proton gradient, which generated heat through uncoupling of oxidative phosphorylation from the electron transport chain (Nicholls and Locke, 1984). Several studies suggest that UCPs might be involved in many metabolic functions, including fatty acid transport, regulation of insulin secretion, and regulation of mitochondrial superoxide generation (Echtay, 2007). UCP2 was suggested to be a negative regulator of insulin secretion and acted through a mild uncoupling mechanism (Chan et al., 1999). Moreover, UCP2 and UCP3 are activated by superoxide from the mitochondrial inner membrane (Echtay et al., 2002; Talbot et al., 2004). It was proposed that UCPs could reduce mitochondrial reactive oxygen species (ROS) generation, and therefore attenuate damage derived from these molecules (Murphy et al., 2003). In a recent study, endothelial UCP2 was shown to function mainly in the control ROS generation in mitochondria during hyperglycemia (Koziel et al., 2015). Moreover, the recently identified UCP4 and UCP5 were specifically localized in the brain, and their roles were suggested to be in the modulation of energy production and mitochondrial ROS levels (Mao et al., 1999; Sanchis et al., 1998; Yu et al., 2000). Ectopic expression of these proteins resulted in lower mitochondrial membrane potential $\left(\psi_{\mathrm{m}}\right)$ and ROS content. It was proposed that high $\psi_{\mathrm{m}}$ is strongly correlated with increases in ROS in mitochondria, and consequently mitochondrial oxidative damage (Boveris et al., 1972; Echtay, 2007; Erlanson-Albertsson, 2003). It was indeed demonstrated that 4-hydroxynonenal (HNE), derived from lipid peroxidation, reduced mitochondrial ROS production, through uncoupling, and decreased $\psi_{\mathrm{m}}$ (Echtay and Brand, 2007; Echtay et al., 2003).

Under restricted diet conditions, UCP2, 4, and 5 levels were shown to increase, which was associated with decreased ROS production and reduced neurodegeneration in models of Parkinson's disease (Duan and Mattson, 1999; Echtay, 2007; Sullivan et al., 2004). It has been suggested that UCPs regulate pathways involved in neurodegeneration, and protect against neurodegenerative diseases and aging through controlling ROS-induced oxidative stress. In addition, it was reported that Ucp2 knockout mice have enhanced resistance to the parasite Toxoplasma, presumably through increased ROS production in macrophages (Arsenijevic et al., 2000).

Surprisingly there are few studies on UCP in Caenorhabditis elegans (C. elegans), despite this species providing a good research model. C. elegans has only one UCP, UCP-4, which is 
a homologue of mammalian UCP4 (Iser et al., 2005). According to a study by Iser et al. (2005), despite elevated ATP levels, there were no significant differences between wild type and ucp-4 mutants in terms of life span, temperature tolerance, and resistance to applied oxidative stress. However, the ucp-4 mutant was sensitive to cold stress. This indicates that $u c p-4$ in $C$. elegans has a role as a thermogenin. In addition, it was suggested that ceUCP4 controls succinate transport to achieve regulation of complex II-mediated oxidative phosphorylation (Pfeiffer et al., 2011). Given that mammals have multiple UCPs with multiple functions, the single $C$. elegans UCP could be multifunctional and involved in several mechanisms. However, its role in neuronal aging and innate immunity is not known. We therefore investigated the role of $C$. elegans UCP4 in neuronal aging and pathogen resistance.

\section{MATERIALS AND METHODS}

\section{Strains and constructs}

Wild type Bristol N2 strain, ucp-4 deletion mutant (ok195), and CZ10175 zdls5 (Pmec-4::gfp) were obtained from the Caenorhabditis Genetics Center (CGC) at the University of Minnesota. The $u c p-4$ deletion mutant was crossed to zdls 5 to obtain ucp-4 (ok195);zdls5 (Pmec-4::gfp). We cultured C. elegans according to published protocols (Brenner, 1974).

To clone the $u c p-4$ promoter region, approximately $1.9 \mathrm{~kb}$ of the $5^{\prime}$-upstream region of $u c p-4$ gene was amplified by polymerase chain reaction (PCR) using genomic DNA from worm lysate as a template. The amplified DNA was inserted into the Fire vector, pPD95.79 to generate Pucp-4::gfp. The amplified $1.6 \mathrm{~kb} u c p-4$ genomic DNA was combined with Pucp-4::gfp to construct Pucp-4::ucp-4::gfp. To obtain ucp-4 overexpression constructs, the amplified $1.6 \mathrm{~kb} u c p-4$ fragment was cloned into the Fire vector, pPD49.83, Phs::ucp-4. Subsequently, the heatshock promoter of the vector was replaced by the $1.9 \mathrm{~kb} u c p-4$ promoter region to generate Pucp-4::ucp-4. Microinjection was performed as previously described (Mello and Fire, 1995) and the plasmid pRF4 containing a dominant gene (rol-6) was coinjected.

For RNAi bacterial feeding constructs, ucp-4 cDNA was obtained by reverse transcription polymerase chain reaction (RTPCR) followed by PCR. The amplified $1 \mathrm{~kb} u c p-4 \mathrm{cDNA}$ was inserted into the Fire feeding vector (L4440). The RNAi construct was introduced to HT115 (DE3) bacteria. Feeding experiments were performed as described by Kamath et al. (2001).

\section{MitoTracker and TMRE experiments}

MitoTracker Red CMXRos (Invitrogen, Life Technologies Corporation, USA) was prepared following the manufacturer's protocol and added to the growth media plate at a final concentration of $2 \mu \mathrm{g} / \mathrm{ml}$. The worms were transferred to the media and incubation proceeded for $16 \mathrm{~h}$.

Tetramethylrhodamine (TMRE, Invitrogen) is a cell-permeable and cationic fluorescent dye that is an indicator of mitochondrial membrane potential $\left(\psi_{\mathrm{m}}\right)$ (Farkas et al., 1989; Loew et al., 1993; Yoneda et al., 2004). TMRE in DMSO (50 $\mu \mathrm{M})$ was applied to the worm plates at a final concentration of $0.1 \mu \mathrm{M}$. Worms were incubated on TMRE plates for $16 \mathrm{~h}$ and then prepared for observation after washing with M9 buffer (Yoneda et al., 2004). For ectopic overexpression experiments, heat shock was performed for $2 \mathrm{~h}$ at $30^{\circ} \mathrm{C}, 12 \mathrm{~h}$ before observation.

Observation of neuronal defects

We scored an individual adult worm as one neuronal defect when it displayed at least one defect such as an outgrowth in the anterior lateral microtubule cells (ALM) or branching, blebbing, and waving in the posterior lateral microtubule cells (PLM) (Cho et al., 2015). All observations were conducted using a fluorescent microscope (80i-DS-Fi1, Nikon). For assessing neuronal defects, animals with ectopic overexpression of target proteins were subjected to a mild heat shock at $25^{\circ} \mathrm{C}$ for $2 \mathrm{~h}$ every $24 \mathrm{~h}$.

\section{Pathogen experiments}

Staphylococcus aureus was cultured overnight at $37^{\circ} \mathrm{C}$ in $\mathrm{BHI}$ media, after which the culture was diluted 1:10 with the same media. This diluted solution $(100 \mu \mathrm{l})$ was spread onto plates and incubated at $37^{\circ} \mathrm{C}$ for $6 \mathrm{~h}$, followed by $6 \mathrm{~h}$ of cooling at $20^{\circ} \mathrm{C}$. For treatment with heat-killed pathogens, plates were incubated at $65^{\circ} \mathrm{C}$ for $6 \mathrm{~h}$ to kill the pathogen. Worms were transferred to prepared media (previously seeded with heatkilled or live pathogens) and the live worms were counted every $6 \mathrm{~h}$ until no living worms remained. Data are presented as mean survival days, which were calculated using the OASIS program (Yang et al., 2011).

\section{RESULTS AND DISCUSSION}

ucp-4 is expressed ubiquitously and UCP-4 is localized in the mitochondria in $C$. elegans

A previous study reported that $u c p-4$ was expressed in the head muscles, pharynx, and body wall muscles of $C$. elegans (Iser et al., 2005). We investigated $u c p-4$ expression and UCP4 localization. Using a transcriptional reporter (Pucp-4::gfp), GFP expression indicated that $u c p-4$ was expressed throughout the body of adult worms and that expression was initiated at an early developmental stage (Figs. $1 \mathrm{~A}$ and $1 \mathrm{~B}$, respectively). $u c p-4$ expression was observed in several tissues, including pharyngeal muscle, body wall muscle, and head sensory neurons (Figs. 1C, 1D, and $1 \mathrm{E}$ respectively). These results suggest that $u c p-4$ was expressed in most tissues throughout the life of the worm. Regarding UCP-4 localization, a translational reporter (Pucp-4::ucp-4::gfp) was used. In UCP-4::GFP transgenic animals, GFP-tagged UCP-4 was specifically localized in the mitochondria of the body wall muscle and hypodermis (Figs. 1F and $1 \mathrm{G}$ ). MitoTracker staining, overlaid with green fluorescence in the hypodermis, suggested that UCP-4 might be located inside the mitochondria, and probably in the inner membrane (Fig. 1H).

ucp-4 mutant reveals increased mitochondrial membrane potential $\left(\psi_{\mathrm{m}}\right)$

It has been suggested that increased mitochondrial membrane potential $\left(\psi_{\mathrm{m}}\right)$ is positively associated with reactive oxygen species (ROS) generation in the mitochondria (Hansford et al., 1997; Korshunov et al., 1997; Votyakova and Reynolds, 2001). Moreover, UCPs dissipate the mitochondrial proton gradient, resulting in decreased $\psi_{\mathrm{m}}$ (Korshunov et al., 1997). To determine if UCP-4 is involved in regulating $\psi_{\mathrm{m}}$, we used TMRE staining to visualize $\psi_{\mathrm{m}}$ in wild type, ucp-4 mutant, and ucp-4 overexpressing animals. As shown in Fig. 2, $\psi_{\mathrm{m}}$ was increased in the $u c p-4$ mutants compared to that of wild type worms (Figs. $2 \mathrm{~A}$ and $2 \mathrm{~B}$, respectively); this result was consistent with previous studies (Iser et al., 2005; Ji et al., 2012). To confirm the involvement of $u c p-4$ in $\psi_{\mathrm{m}}, u c p-4$ was overexpressed using a heat shock promoter, Phs::ucp-4, and the worms were stained by TMRE. TMRE intensity in overexpressing animals was lowest among all strains (Fig. 2D). Wild type animals with the heat 

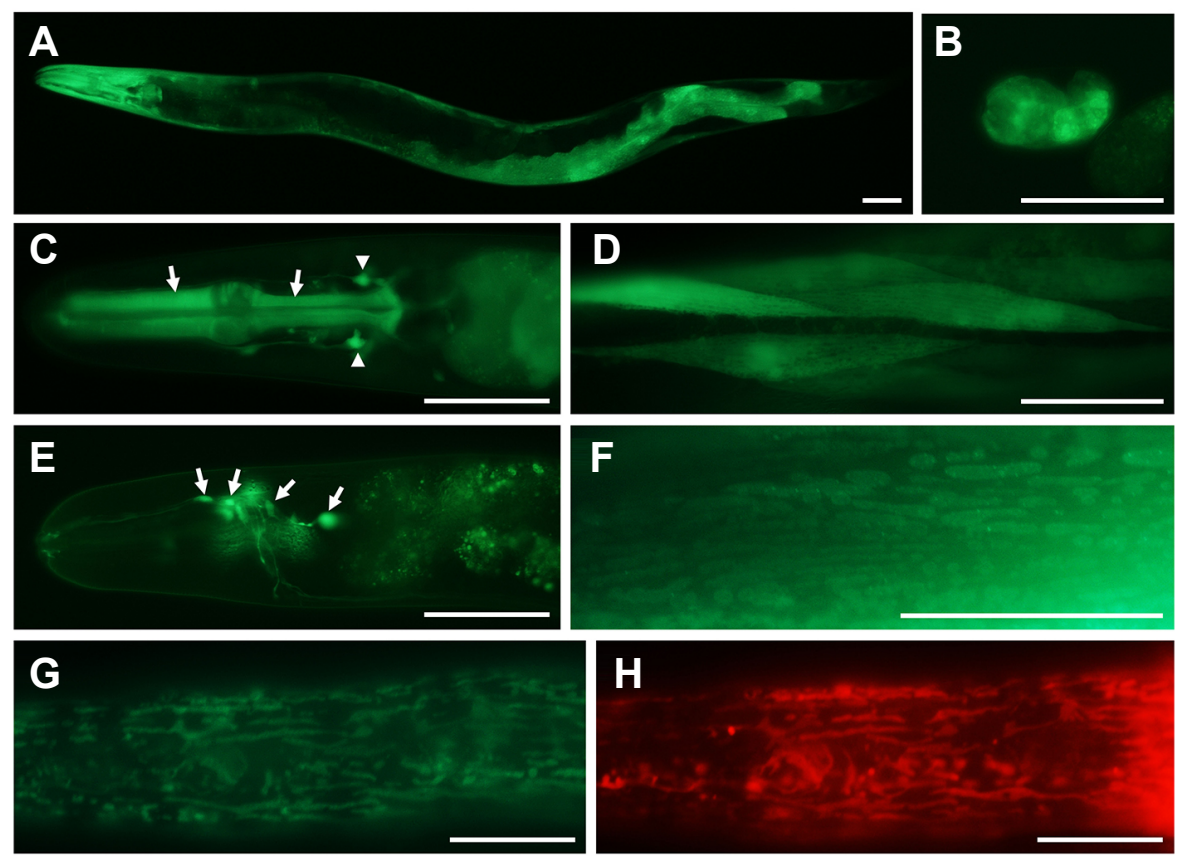

Fig. 1. ucp-4 expression and UCP-4 localization in $C$. elegans. ucp-4 was expressed throughout the entire body (A) and in a comma stage embryo (B) in the transgenic animal carrying Pucp-4::gfp. Higher magnification showed intense expression of $u c p-4$ in pharyngeal muscle and head neurons $(\mathrm{C}$, arrows and arrowheads, respectively), body wall muscle (D), and head neurons (E, arrows). UCP-4 was localized in the body wall muscles and in the hypodermis ( $F$ and $G$ respectively) in the transgenic worm carrying the Pucp-4::ucp-4::gfp construct. (H) The same hypodermis image in $\mathrm{G}$ stained with MitoTracker is presented. Scale bars $=50 \mu \mathrm{m}$.
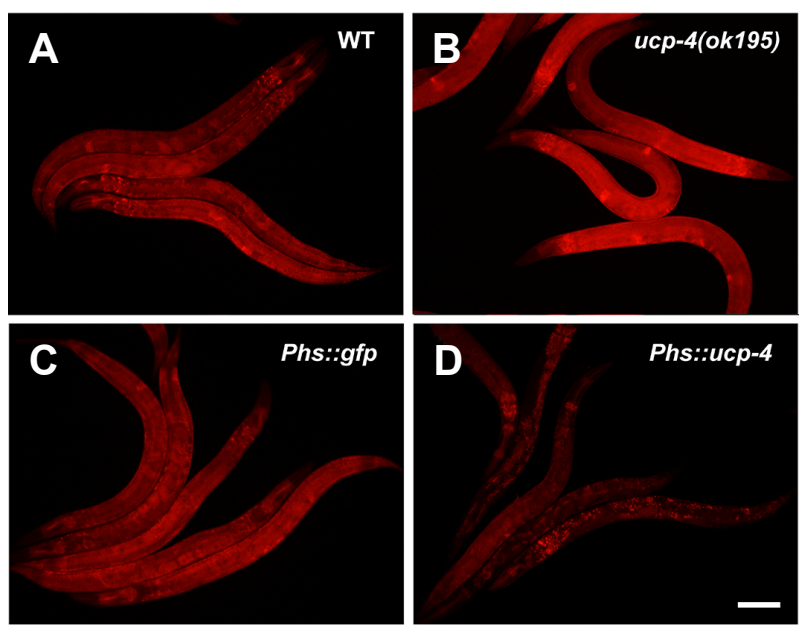

Fig. 2. The $u c p-4$ mutant shows increased mitochondrial membrane potential $\left(\psi_{\mathrm{m}}\right) . \psi_{\mathrm{m}}$ was assessed by TMRE. WT, wild type (A); ucp-4, ucp-4 mutant (B); Phs::gfp (C); Phs::ucp-4, ucp-4 overexpression under control of the heat shock promoter (D). Scale bar $=$ $100 \mu \mathrm{m}$.

shock promoter showed no difference in TMRE intensity compared to wild type animals; therefore, the heat shock promoter itself did not affect $\psi_{\mathrm{m}}$ (Fig. 2C). Low $\psi_{\mathrm{m}}$ in ucp-4 overexpressing worms could be the result of proton gradient dissipation. Therefore, UCP-4 could lead to low energy efficiency and low ROS levels.

ucp-4 mutant displays increased neuronal defects Increased ROS, probably in the context of oxidative damage, is correlated with neuronal diseases such as Parkinson's and
Alzheimer's (Fahn and Cohen, 1992; Lin and Beal, 2006). Human UCP2 was shown to protect neurons against oxidative stress (Mattiasson and Sullivan, 2006). We therefore examined if UCP-4 is involved in neuronal defects during the aging process. In recent reports, mechanosensory touch receptor neurons, anterior lateral microtubule cells (ALM) and posterior lateral microtubule cells (PLM), showed neuronal degenerations during aging, which included soma outgrowth, axon blebbing, and wavy processes (Pan et al., 2011; Tank et al., 2011; Toth et al., 2012). In addition, ALM and PLM cells are well-studied and easily observed (Chen et al., 2013). Therefore, We used the zdls5 (Pmec-4::gfp) background to observe and score ALMs and PLMs during aging. Imaging of touch receptor neurons in zdls5 worms showed ALM and PLM cells on day 1 (Fig. 3A). Fig. 3B shows standard ALM outgrowth and PLM wavy defects in the 10-day control animal. On day 15, severe ALM outgrowth and PLM waving and blebbing phenotypes were observed as aging progressed in control worms (Figs. 3C, 3D, and 3E).

To understand $u c p-4$ function in neuronal aging, $u c p-4$ deficient and $u c p-4$ overexpressing worms were examined. First, both $u c p-4$ deletion mutants and $u c p-4$ RNAi treated animals were used for neuronal observation during aging to determine whether effects are the result of $u c p-4$ itself. For RNAi treatment in worms, ucp-4 RNAi was applied to zdls5 animals (Figs. 3F and $3 G$ ). Figures $3 F$ and $3 G$ show that ALM and PLM defects progressed in an age-dependent manner regardless of the presence of $u c p-4$ (comparing day 1 to day 15). For example, ALMs in the 15-day control showed a $67 \%$ defect, which was much greater than the $14 \%$ defect in 1-day animals (Fig. 3F). Moreover, the ucp-4 mutant showed markedly increased ALM defects, specifically, $22 \%$ on day 1 and $89 \%$ on day 15 (Fig. 3F). Animals treated with ucp-4 RNAi showed similar results to those of the $u c p-4$ mutant.

For PLMs, neuronal defects gradually increased with aging in control and ucp-4 deficient animals (Fig. 3G). However, PLM waving phenotypes were much more severe in $u c p-4$ mutants 

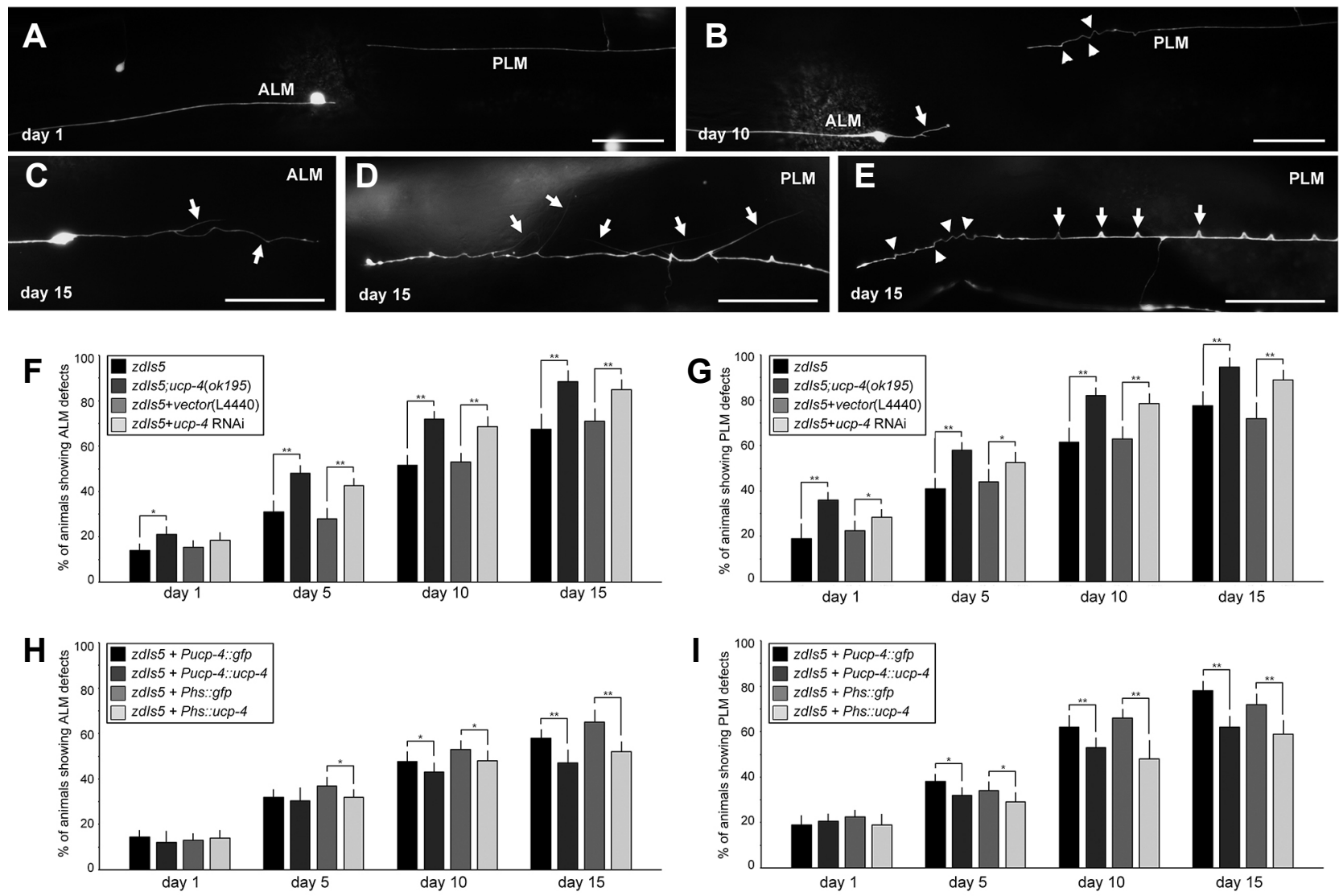

Fig. 3. Neuronal defects are increased in the ucp-4 mutant. (A-E) A representative image of a mechanosensory neuron from a control worm is presented on day $1(A), 10(B)$, and $15(C, D$, and $E)$. An arrow indicates ALM outgrowth and arrowheads indicate PLM waving in B. Arrows show ALM outgrowth in C; arrows indicate PLM branching in D. Arrows indicate PLM blebbing and arrowheads show PLM waving in E. Scale bars $=50 \mu \mathrm{m}$. (F and H) ALM neuronal defects in the ucp-4 mutant and in ucp-4 RNAi treated worms (F) and ucp-4 overexpressing animals $(\mathrm{H})$ are presented as a percentage relative to that of control animals. (G and I) PLM neuronal defects in the ucp-4 mutant and in ucp-4 RNAi treated worms $(\mathrm{G})$ and ucp-4 overexpressing animals $(\mathrm{I})$ are presented as a percentage relative to that of the control. zdls5, zdls5 (Pmec4::gfp); zdls5;ucp-4 (ok195), ucp-4 in zdls5; zdls5 + vector (L4440), empty vector (L4440) in zdls5; zdls5 + ucp-4 RNAi, zdls5 treated with ucp4 RNAi (F and G). zdls5 + Pucp-4::gfp, Pucp-4::gfp in zdls5; zdls5 + Pucp-4::ucp-4, Pucp-4::ucp-4 in zdls5; zdls5 + Phs::gfp, Phs::gfp in zdls5; zdls5 + Phs::ucp-4, Phs::ucp-4 in zdls5 (H and I) $\mathrm{n}>100$ worms per line in each experiment. Four independent experiments were performed. Error bars represent SD. ${ }^{*} p<0.05 ;{ }^{\star *} p<0.01$, based on a Student's $t$-test.

compared to that of control worms (Fig. 3G). Quantification indicated that both $u c p-4$ mutants and $u c p-4$ RNAi treated worms resulted in more neuronal defects compared to that in controls at all time points tested (Figs. 3F and 3G). These results suggest that only ucp-4 contributes to neuronal defects during aging. For confirmation, $u c p-4$ overexpressing animals were also studied during aging. Pucp-4::ucp-4 and Phs::ucp-4 constructs were injected into $z d / s 5$ for observation in overexpressing worms (Figs. $3 \mathrm{H}$ and $3 \mathrm{I}$ ). On day 1, ALM and PLM phenotypes were not significantly different between controls (Phs:::gfp) and ucp-4 overexpressing animals (Pucp-4::ucp-4 and Phs::ucp-4). As aging progressed, all animals showed a gradual increase in neuronal defects (Figs. $3 \mathrm{H}$ and $3 \mathrm{I}$ ). However, both ucp-4 overexpressing animals (Pucp-4::ucp-4 and Phs::ucp-4) consistently presented a lower number of defects compared to that in either group of control animals (Pucp-4::gfp and Phs::gfp) (Figs. $3 \mathrm{H}$ and $3 \mathrm{I}$ ). These results led us to conclude that UCP-4 attenuates neuronal defects during aging, probably by lowering the mitochondrial membrane potential $\left(\psi_{\mathrm{m}}\right)$ and consequently lowering ROS levels.

According to the free radical theory, ROS might cause aging and aging-related disease (Harman, 1972). Mild uncoupling by UCPs resulting in lowered ROS levels can alleviate aging and aging-associated diseases such as Parkinson's and Alzheimer's disease that are driven by oxidative damage. However this has not yet been clearly proven (Beckman and Ames, 1998; Divakaruni and Brand, 2011). The quantification of a mild uncoupling effect requires the development of specific methods to measure physiological ROS content, especially in native conditions (Divakaruni and Brand, 2011). Despite technical difficulty, the application of mild uncoupling has shown beneficial results. For example, mice expressing muscle-specific UCP1 showed decreased adiposity, increased metabolic rate, and diminished age-related diseases (Gates et al., 2007). Ucp2 overexpressing mice showed neuronal protection after global ischemia and after transient focal ischemia (Deierborg et al., 

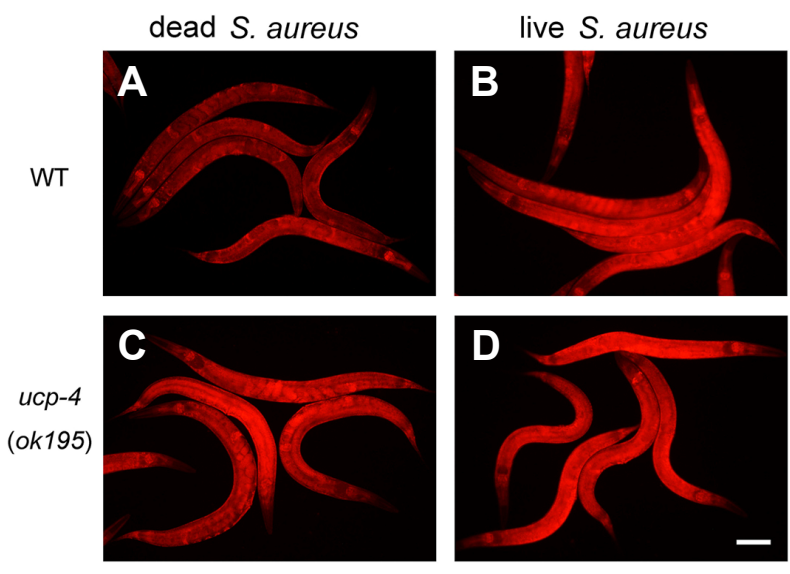

$\mathbf{E}$

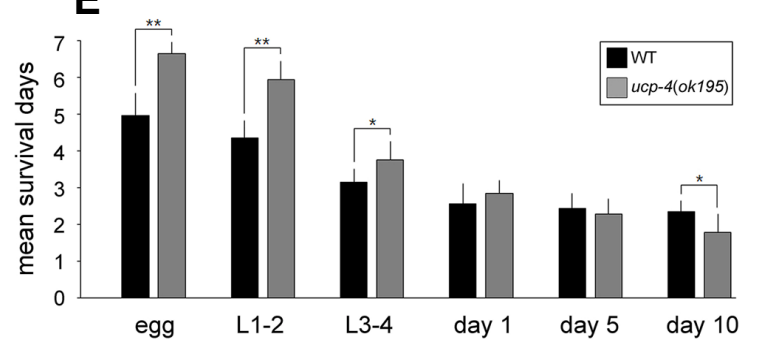

Fig. 4. Deficiency of UCP-4 results in resistance to $S$. aureus. Mitochondrial membrane potential $\left(\psi_{\mathrm{m}}\right)$ was measured by TMRE after wild type worms were fed heat-killed or live $S$. aureus (A and B, respectively). (C) and (D) represent TMRE stained ucp-4 mutants feeding on either heat-killed or live $S$. aureus, respectively. Scale bar $=100 \mu \mathrm{m}$. (E) Mean survival days of the wild type and the ucp-4 mutant after exposure to pathogen-seeded plates. Each experimental result was calculated by OASIS. The average of five experimental replicates was plotted. $n>300$ worms per line in each experiment. WT, wild type; ucp-4, ucp-4 mutant; egg, animals were exposed to the pathogen at the egg stage; L1-2, at L12 stage; L3-4, at L3-4 stage; adult day 1, 5, and 10, at day 1, 5, and 10 , respectively. Error bars $=\mathrm{SD} .{ }^{*} p<0.05 ;{ }^{* *} p<0.01$, based on a Student's $t$-test.

2008; Haines and Li, 2012) Moreover, our results suggest that ucp-4 overexpression in C. elegans alleviates neuronal defects that occur during aging. Therefore, UCPs can regulate $\psi_{\mathrm{m}}$, which protects neurons during aging. Proper application of mild uncoupling might be useful to treat metabolic or age-related diseases. Further studies are required to establish mild uncoupling and determine how it is regulated during age-related neurodegeneration.

The ucp-4 mutant is resistant to the pathogen $S$. aureus Increased ROS can cause oxidative damage, especially in the mitochondria (Harman, 1956). However, an increase in ROS can be beneficial for innate immunity as a major defense system against pathogens (Arsenijevic et al., 2000; Basu Ball et al., 2011). Moreover, in C. elegans, increased ROS levels were shown to occur in the intestine at the site of pathogen infection (Chavez et al., 2007). Because UCP-4 is involved in regulating mitochondrial membrane potential $\left(\psi_{\mathrm{m}}\right)$, we investigated the function of UCP-4 in the response to pathogens. First, we compared $\psi_{\mathrm{m}}$ using TMRE staining in wild types and in ucp-4 mu- tants upon exposure to either live (pathogenic) or heat-killed (non-pathogenic) Staphylococcus aureus (S. aureus). As seen in Fig. 4, the $u c p-4$ mutant had high $\psi_{m}$ compared to that of the wild type without pathogen exposure (Figs. 4A and $4 C$, respectively). After pathogen exposure, there was no significant change in $\psi_{\mathrm{m}}$ between live and heat-killed pathogen exposed ucp-4 mutants (Fig. 4D). In contrast, wild type worms showed an increase in $\psi_{\mathrm{m}}$ upon exposure to live pathogens (Fig. 4B).

This increased $\psi_{\mathrm{m}}$ could be related to pathogen resistance; we therefore assayed worm survival. We placed animals on pathogen-seeded plates during each developmental stage, specifically egg, L1-2, L3-4, and adult day 1, 5, and 10. After the worms were transferred, surviving animals were counted each day until all were dead. We used the OASIS program to calculate mean survival days based on the raw data (Yang et al., 2011). As seen in Fig. 4E, the ucp-4 mutant survived longer than the wild type when exposed to the pathogen at the egg stage, L1-2, L3-4, and the young adult stage (day 1). However, exposure at the aged adult stages (days 5 and 10) produced different results. Specifically, the ucp-4 mutants were more susceptible to the pathogen compared to wild type at this stage.

Considering the increase in $\psi_{\mathrm{m}}$ in wild type animals upon pathogen exposure, the resulting increase in ROS could serve to initiate an immune response. However, the ucp-4 mutant displayed high ROS levels without the presence of the pathogen, which could account for the observed resistance. As shown in Fig. 4, ucp-4 mutants exposed to pathogens at an early stage showed increased resistance, but were susceptible when exposed to the pathogen in an aged adult stage. This inconsistency between young and aged animals might be due to the free radical aging process and/or gradual ROS response (Hekimi et al., 2011). It was shown that silencing UCP2 in macrophages resulted in increased ROS production that could kill the pathogen via direct oxidative damage. As a result, the UCP2 knocked-down macrophage showed enhanced resistance to the Leishmania parasite (Basu Ball et al., 2011). When young ucp-4 mutants were exposed to the pathogen, the increased ROS could function as microbicidal molecules. In addition, increased ROS could facilitate an immune responseduring pathogen attack. Indeed, a recent study showed that moderately increased ROS in the mitochondria enhances immunity against pathogens via a feedback mechanism involving hypoxia-inducible factor 1 (HIF-1) and AMP-activated protein kinase (AMPK) in C. elegans (Hwang et al., 2014).

Alternatively, high levels of ROS can cause oxidative damage. It was observed that various organisms accumulated oxidative damage of macromolecules such as DNA, proteins, and lipids during aging (Back et al., 2012; Harman, 1956; 1972; 2009; Sohal and Weindruch, 1996). When ucp-4 mutants aged, their macromolecules could be progressively damaged by increased ROS. The cumulative damage via ROS and aging in the ucp-4 mutant could cause susceptibility to the pathogen. ROS increases can thus function in two opposite ways. The initial ROS increase against a pathogen attack could be beneficial to kill the pathogen and trigger an immune response. However, the constant high level of ROS could cause deterioration of structures consisting of physical barriers to infection by pathogen. Since ROS can exert both positive and negative responses, the regulation of ROS levels could be critical for innate immunity. This dual role is probably tightly linked to ROS balance; therefore, further studies are necessary to elucidate how the ROS balance in the innate immune system is sensed and regulated during pathogen exposure. 


\section{ACKNOWLEDGMENTS}

We thank the Caenorhabditis Genetics Center (CGC) at the University of Minnesota, USA, for the worm strains and Andrew Fire for the Fire vectors. The study was supported by a research fund from Chosun University, 2011.

\section{REFERENCES}

Arsenijevic, D., Onuma, H., Pecqueur, C., Raimbault, S., Manning, B.S., Miroux, B., Couplan, E., Alves-Guerra, M.C., Goubern, M., Surwit, R., et al. (2000). Disruption of the uncoupling protein-2 gene in mice reveals a role in immunity and reactive oxygen species production. Nat. Genet. 26, 435-439.

Back, P., Braeckman, B.P., and Matthijssens, F. (2012). ROS in aging Caenorhabditis elegans: damage or signaling? Oxid. Med. Cell Longev. 2012, 608478

Basu Ball, W., Kar, S., Mukherjee, M., Chande, A.G., Mukhopadhyaya, R., and Das, P.K. (2011). Uncoupling protein 2 negatively regulates mitochondrial reactive oxygen species generation and induces phosphatase-mediated anti-inflammatory response in experimental visceral leishmaniasis. J. Immunol. 187, 1322-1332.

Beckman, K.B., and Ames, B.N. (1998). The free radical theory of aging matures. Physiol. Rev. 78, 547-581.

Boveris, A., Oshino, N., and Chance, B. (1972). The cellular production of hydrogen peroxide. Biochem. J. 128, 617-630.

Brenner, S. (1974). The genetics of Caenorhabditis elegans. Genetics 77, 71-94.

Chan, C.B., MacDonald, P.E., Saleh, M.C., Johns, D.C., Marban, E., and Wheeler, M.B. (1999). Overexpression of uncoupling protein 2 inhibits glucose-stimulated insulin secretion from rat islets. Diabetes 48, 1482-1486.

Chavez, V., Mohri-Shiomi, A., Maadani, A., Vega, L.A., and Garsin, D.A. (2007). Oxidative stress enzymes are required for DAF-16mediated immunity due to generation of reactive oxygen species by Caenorhabditis elegans. Genetics 176, 1567-1577.

Chen, C.H., Chen, Y.C., Jiang, H.C., Chen, C.K., and Pan, C.L. (2013). Neuronal aging: learning from C. elegans. J. Mol. Signal. 8, 14.

Cho, I., Hwang, G.J., and Cho, J.H. (2015). pxn-1 and pxn-2 May Interact Negatively during Neuronal Development and Aging in C. elegans. Mol. Cells 38, 729-733.

Deierborg, T., Wieloch, T., Diano, S., Warden, C.H., Horvath, T.L., and Mattiasson, G. (2008). Overexpression of UCP2 protects thalamic neurons following global ischemia in the mouse. J. Cereb. Blood Flow Metab. 28, 1186-1195.

Divakaruni, A.S., and Brand, M.D. (2011). The regulation and physiology of mitochondrial proton leak. Physiology (Bethesda) 26, 192-205

Duan, W., and Mattson, M.P. (1999). Dietary restriction and 2deoxyglucose administration improve behavioral outcome and reduce degeneration of dopaminergic neurons in models of Parkinson's disease. J. Neurosci. Res. 57, 195-206.

Echtay, K.S. (2007). Mitochondrial uncoupling proteins--what is their physiological role? Free Radic. Biol. Med. 43, 1351-1371.

Echtay, K.S., and Brand, M.D. (2007). 4-hydroxy-2-nonenal and uncoupling proteins: an approach for regulation of mitochondrial ROS production. Redox Rep. 12, 26-29.

Echtay, K.S., Murphy, M.P., Smith, R.A., Talbot, D.A., and Brand, M.D. (2002). Superoxide activates mitochondrial uncoupling protein 2 from the matrix side. Studies using targeted antioxidants. J. Biol. Chem. 277, 47129-47135.

Echtay, K.S., Esteves, T.C., Pakay, J.L., Jekabsons, M.B., Lambert, A.J., Portero-Otin, M., Pamplona, R., Vidal-Puig, A.J., Wang, S., Roebuck, S.J., et al. (2003). A signalling role for 4-hydroxy-2nonenal in regulation of mitochondrial uncoupling. EMBO J.22, 4103-4110.

Erlanson-Albertsson, C. (2003). The role of uncoupling proteins in the regulation of metabolism. Acta. Physiol. Scand. 178, 405-412.

Fahn, S., and Cohen, G. (1992). The oxidant stress hypothesis in Parkinson's disease: evidence supporting it. Ann. Neurol. 32, 804-812.

Farkas, D.L., Wei, M.D., Febbroriello, P., Carson, J.H., and Loew, L.M. (1989). Simultaneous imaging of cell and mitochondrial membrane potentials. Biophys. J. 56, 1053-1069.

Gates, A.C., Bernal-Mizrachi, C., Chinault, S.L., Feng, C., Schneider, J.G., Coleman, T., Malone, J.P., Townsend, R.R., Chakravarthy, M.V., and Semenkovich, C.F. (2007). Respiratory uncoupling in skeletal muscle delays death and diminishes agerelated disease. Cell Metab. 6, 497-505

Haines, B., and Li, P.A. (2012). Overexpression of mitochondrial uncoupling protein 2 inhibits inflammatory cytokines and activates cell survival factors after cerebral ischemia. PLoS One 7, e31739.

Hansford, R.G., Hogue, B.A., and Mildaziene, V. (1997). Dependence of $\mathrm{H} 2 \mathrm{O} 2$ formation by rat heart mitochondria on substrate availability and donor age. J. Bioenerg. Biomembr. 29, 89-95.

Harman, D. (1956). Aging: a theory based on free radical and radiation chemistry. J. Gerontol. 11, 298-300.

Harman, D. (1972). The biologic clock: the mitochondria? J. Am. Geriatr. Soc. 20, 145-147.

Harman, D. (2009). Origin and evolution of the free radical theory of aging: a brief personal history, 1954-2009. Biogerontology 10, 773-781.

Hekimi, S., Lapointe, J., and Wen, Y. (2011). Taking a "good" look at free radicals in the aging process. Trends Cell Biol. 21, 569-576.

Hwang, A.B., Ryu, E.A., Artan, M., Chang, H.W., Kabir, M.H., Nam, H.J., Lee, D., Yang, J.S., Kim, S., Mair, W.B., et al. (2014). Feedback regulation via AMPK and HIF-1 mediates ROSdependent longevity in Caenorhabditis elegans. Proc. Natl. Acad. Sci. USA 111, E4458-4467.

Iser, W.B., Kim, D., Bachman, E., and Wolkow, C. (2005) Examination of the requirement for ucp-4, a putative homolog of mammalian uncoupling proteins, for stress tolerance and longevity in C. elegans. Mech. Ageing Dev. 126, 1090-1096.

Ji, C., Guo, W., Zhang, M., Lu, X., Ni, Y., and Guo, X. (2012). Caenorhabditis elegans ucp-4 regulates fat metabolism: suppression of ucp-4 expression induced obese phenotype and caused impairment of insulin like pathway. Gene 491, 158-164.

Kamath, R.S., Martinez-Campos, M., Zipperlen, P., Fraser, A.G., and Ahringer, J. (2001). Effectiveness of specific RNA-mediated interference through ingested double-stranded RNA in Caenorhabditis elegans. Genome Biol 2, 1-10.

Korshunov, S.S., Skulachev, V.P., and Starkov, A.A. (1997). High protonic potential actuates a mechanism of production of reactive oxygen species in mitochondria. FEBS Lett. 416, 15-18.

Koziel, A., Sobieraj, I., and Jarmuszkiewicz, W. (2015). Increased activity of mitochondrial uncoupling protein 2 improves stress resistance in cultured endothelial cells exposed in vitro to high glucose levels. Am. J. Physiol. Heart Circ. Physiol. 309, H147156.

Lin, M.T., and Beal, M.F. (2006). Mitochondrial dysfunction and oxidative stress in neurodegenerative diseases. Nature $443,787-$ 795.

Loew, L.M., Tuft, R.A., Carrington, W., and Fay, F.S. (1993). Imaging in five dimensions: time-dependent membrane potentials in individual mitochondria. Biophys. J. 65, 2396-2407.

Mao, W., Yu, X.X., Zhong, A., Li, W., Brush, J., Sherwood, S.W., Adams, S.H., and Pan, G. (1999). UCP4, a novel brain-specific mitochondrial protein that reduces membrane potential in mammalian cells. FEBS Lett. 443, 326-330.

Mattiasson, G., and Sullivan, P.G. (2006). The emerging functions of UCP2 in health, disease, and therapeutics. Antioxid. Redox Signal. 8, 1-38.

Mello, C., and Fire, A. (1995). DNA transformation. Methods Cell Biol 48, 451-482.

Murphy, M.P., Echtay, K.S., Blaikie, F.H., Asin-Cayuela, J., Cocheme, H.M., Green, K., Buckingham, J.A., Taylor, E.R., Hurrell, F., Hughes, G., et al. (2003). Superoxide activates uncoupling proteins by generating carbon-centered radicals and initiating lipid peroxidation: studies using a mitochondria-targeted spin trap derived from alpha-phenyl-N-tert-butylnitrone. J. Biol. Chem. 278, 48534-48545.

Nicholls, D.G., and Locke, R.M. (1984). Thermogenic mechanisms in brown fat. Physiol. Rev. 64, 1-64.

Pan, C.L., Peng, C.Y., Chen, C.H., and McIntire, S. (2011). Genetic analysis of age-dependent defects of the Caenorhabditis elegans touch receptor neurons. Proc. Natl. Acad. Sci. USA 108, 92749279. 
Pfeiffer, M., Kayzer, E.B., Yang, X., Abramson, E., Kenaston, M.A., Lago, C.U., Lo, H.H., Sedensky, M.M., Lunceford, A., Clarke, C.F., et al. (2011). Caenorhabditis elegans UCP4 protein controls complex II-mediated oxidative phosphorylation through succinate transport. J. Biol. Chem. 286, 37712-37720.

Sanchis, D., Fleury, C., Chomiki, N., Goubern, M., Huang, Q., Neverova, M., Gregoire, F., Easlick, J., Raimbault, S., LeviMeyrueis, C., et al. (1998). BMCP1, a novel mitochondrial carrier with high expression in the central nervous system of humans and rodents, and respiration uncoupling activity in recombinan yeast. J. Biol. Chem. 273, 34611-34615.

Sohal, R.S., and Weindruch, R. (1996). Oxidative stress, caloric restriction, and aging. Science $273,59-63$.

Sullivan, P.G., Rippy, N.A., Dorenbos, K., Concepcion, R.C., Agarwal, A.K., and Rho, J.M. (2004). The ketogenic diet increases mitochondrial uncoupling protein levels and activity. Ann. Neurol. 55, 576-580.

Talbot, D.A., Lambert, A.J., and Brand, M.D. (2004). Production of endogenous matrix superoxide from mitochondrial complex leads to activation of uncoupling protein 3. FEBS Lett. 556, 111115.

Tank, E.M., Rodgers, K.E., and Kenyon, C. (2011). Spontaneous age-related neurite branching in Caenorhabditis elegans. J.
Neurosci. 31, 9279-9288.

Toth, M.L., Melentijevic, I., Shah, L., Bhatia, A., Lu, K., Talwar, A. Naji, H., Ibanez-Ventoso, C., Ghose, P., Jevince, A., et al. (2012). Neurite sprouting and synapse deterioration in the aging Caenorhabditis elegans nervous system. J. Neurosci. 32, 87788790.

Votyakova, T.V., and Reynolds, I.J. (2001). DeltaPsi(m)-Dependent and -independent production of reactive oxygen species by rat brain mitochondria. J. Neurochem. 79, 266-277.

Yang, J.S., Nam, H.J., Seo, M., Han, S.K., Choi, Y., Nam, H.G., Lee, S.J., and Kim, S. (2011). OASIS: online application for the survival analysis of lifespan assays performed in aging research. PLoS One 6, e23525.

Yoneda, T., Benedetti, C., Urano, F., Clark, S.G., Harding, H.P., and Ron, D. (2004). Compartment-specific perturbation of protein handling activates genes encoding mitochondrial chaperones. J. Cell Sci. 117, 4055-4066.

Yu, X.X., Mao, W., Zhong, A., Schow, P., Brush, J., Sherwood, S.W. Adams, S.H., and Pan, G. (2000). Characterization of novel UCP5/BMCP1 isoforms and differential regulation of UCP4 and UCP5 expression through dietary or temperature manipulation. FASEB J. 14, 1611-1618. 